\title{
Return handling options and order quantities for single period products
}

\author{
D. Vlachos* \\ R. DEKKER ${ }^{\dagger}$
}

8 September 2000

\begin{abstract}
Products which are sold through E-commerce or mail sales catalogues tend to have a much higher return rate than traditional products. The returns are especially problematic for seasonal products. To support decision making in these situations we study various options, which may be considered as strategic decisions, on handling the increased return flow. Closed form analytic expressions for optimal order quantities are obtained by solving the models developed for each option. Decision making guidelines on choosing between return options and some properties of the optimal solutions are presented. We also discuss estimation of the serviceable return rate in practical situations.
\end{abstract}

Keywords : Purchasing, Reverse logistics, E-commerce, single period products

\section{ECONOMETRIC INSTITUTE RESEARCH REPORT EI2000-29/A}

\section{INTRODUCTION}

Over the last decades mail sales (first through catalogues and now through the internet) hold a considerable share in total sales of industrial/commercial business. Moreover, taking into consideration the boom in E-commerce, this share will be further increased. Although there are a lot of similarities between classical sales and mail sales, there are also some major differences, the most important of them being the returns. According to the legislation in many countries the customer has the right to return a product acquired through mail sales and the vendor is obliged to accept it if it is in a good condition, and to pay back its price. The consequences of this customer's option are considerable return flows, which in some cases may be as high as 35\% of the initial orders (Gentry, 1999) (Meyer, 1999). Handling such return flows, mainly consisting of products that are or may easily be brought into an as-good-as-new condition, is an interesting problem, both for regular and single period products. We concentrate on single period (style) products, which have a specific life time (selling period). As style products we consider not only the products that can only be sold during a single period, but also seasonal products with a very short selling period which cannot be stocked till the next period due to high holding costs or obsolescence. The problem is more complex for style products because returns may also be used to satisfy new demand, but only if they are available

\footnotetext{
*Erasmus University Rotterdam, Econometric Institute, The Netherlands and Aristotle University of Thessaloniki, Department of Mechanical Engineering, Greece, email: vlachos@few.eur.nl

$\dagger$ †rasmus University Rotterdam, Econometric Institute, Burg. Oudlaan 50, P.O. box 1738, 3000 DR, The Netherlands, email: rdekker@few.eur.nl.
} 
before the end of the selling period. Otherwise, returns can only be disposed of or sold at a lower price in a secondary market.

The common practice for single period products without returns is the placement of an initial order which arrives before the beginning of the period; the size of this order is given by the classical newsboy formula. Returns may act as a secondary supply channel depending on the already satisfied demand, but only part of its capacity is available due to the fixed length of the selling period. Moreover, to estimate which part of the returns may serve new orders, we have to know not only the total demand of the period, but also its distribution in time. Thus, it is obvious that the classical newsboy optimal order quantity cannot be used here, especially when the return rate is significant.

In this paper we extend the classical newsboy problem, which is to set the initial order quantity for a single period product, to incorporate returns. The remainder of the paper is organized as follows: A short overview of the related literature is presented in section 2 . The problem to determine the initial order quantity of a newsboy type product with returns is modeled and solved in section 3 for various return handling policies. Section 4 provides guidelines on decision making about return policies. Some interesting insights resulting from numerical investigation are presented in section 5. The estimation of the serviceable return flow is discussed in section 6 . Conclusions and extensions are briefly discussed in section 7 .

\section{Literature ReVieW}

The literature related to the classical single period (newsboy) problem is very wide. A lot of extensions have been proposed to fit in various real life situations. Khouja (1999) and Silver et al. (1998) have recently conducted literature reviews, classifying the classical problem extensions proposed. In this short review we concentrate only in papers that have some relation with our research.

A first group of papers addresses the demand forecasting problem of one period products. Various, mainly subjective, methods have been proposed for making interval estimates of demand. These, however, tend to be very inaccurate, with errors of $\pm 50 \%$ being typical rather than exceptional, which makes the determination of initial order sizes hazardous (Chambers and Eglese, 1986). The accuracy of the estimates may be improved by taking into consideration actual demand data from the beginning of the period (Chang and Fyffe, 1971) (Murray and Silver, 1966). But of course such improved estimations only have practical use if a mid-period ordering option exists, which is not the case in our problem.

A second group of papers studies the single period problem considering a second ordering (emergency) option during or in the end of the period (Khouja, 1996) (Lau and Lau, 1997a, b) (Fisher and Raman, 1996). Various models have been proposed to estimate the mid-period order quantity, which is one more decision variable in all these papers. The major difference between these models and our research is that in our case 
the return flow, which acts as a supplementary supply source, depends on the satisfied orders and therefore it is uncontrollable.

A third group of papers investigates the newsboy problem with random yield of the order quantity (Ehrhardt and Taube, 1987) (Noori and Keller, 1986). These papers assume that the replenishment quantity is a random variable different from the initial order quantity and provide analytical expressions for the initial order quantity for specific distributions of the replenishment quantities.

Another group of papers examines return policies of perishable products from retailers to the manufacturer (Webster and Weng, 2000), (Emmons and Gilbert, 1998). Both papers assume a repurchase price for returns and provide optimality conditions for the retail price that maximizes the profit of the retailer. These papers study the problem of supply chain coordination and pricing rather than the problem of setting optimal order quantities for a specific demand.

Finally, a fifth group of papers deals with the supply chain of recoverable products known under the term 'reverse logistics'. Most of these papers study the returns of used products, which are generally not in a good condition and therefore need major recovery or remanufacturing. The returns from mail sales are normally in a quite good condition and they need minor or no recovery to be as-good-as-new. Fleischmann et al. (1997) and Dekker et al. (2000) have carried out reviews on quantitative models for reverse logistics and joint manufacturing and remanufacturing respectively. Although, no direct reference is made to single period products, some interesting remarks can also be found in Van der Laan et al.(1996) and Guide and Srivastava (1998).

In our paper there is a combination of reverse logistic issues (returns) in the classical single period problem, which has not been addressed in the past. Although there are some similarities with some of the above mentioned papers, we believe that the contribution of this paper is to provide simple optimality conditions for the order quantity for some variations of the problem, which have practical value.

\section{SYSTEM DESCRIPTION AND ANALYSIS}

\subsection{System operation}

- We consider a single-period product, for which a significant part of the customer orders are returned to the vendor. The returned products are or can be in as-good-as-new condition and thus it is possible to reuse them to satisfy new orders. We assume that a single ordering decision is to be made before the beginning of the period so as to maximize expected total profit.

The operation of a single-period inventory system with returns is explained through Figure 1, in which cumulative flows of orders, sales and returns are depicted. In Figure 1, the solid black line represents the cumulative demand (customer orders), which receives its maximum value $Q_{m}$ at the end of the selling period 
denoted by $T$. The sales, which are the part of the satisfied demand finally accepted and paid by the customers, are represented by the dashed line. The maximum value for sales $S_{m}$ is obtained only if we are able to satisfy the maximum demand $Q_{m}$.

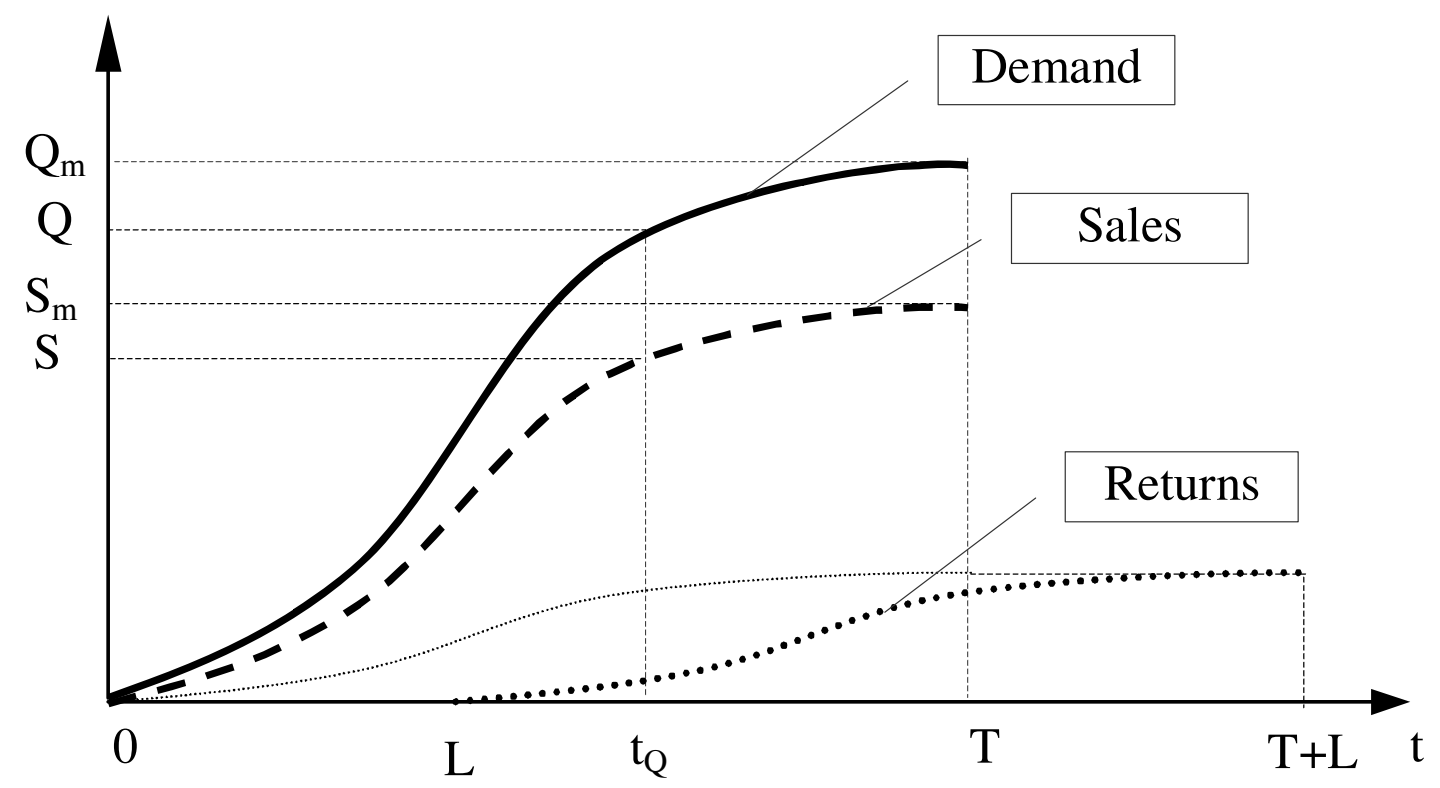

Figure 1: Cumulative orders, sales and returns during $\mathrm{T}$

If the order quantity in the beginning of the period is $Q$, we can only satisfy orders till time $t_{Q}$ and thus, the cumulative sales will be $S$. The returns may be used to satisfy more orders (after time $t_{Q}$ ) and eventually to increase sales up and to $S_{m}$. But, in most practical cases the returns are not available immediately. First, the delivery to the customer takes some time. Then, the customer also needs some time to decide if the product is suitable for him and finally, collection from the customer also takes time. Moreover, for some products some recovery (inspection, packing, minor repair, etc.) must be carried out to bring the product in as-good-as-new condition. The sum of these time delays is denoted by $L$ and it is called the collection and recovery time. So, the returns, which are represented by the dotted line in Figure 1, have a time lag $L$ from the orders. It is obvious that the returns after time $T$ cannot be used to satisfy demand since the demand after the end of the selling period is zero.

\subsection{Return handling options}

- Figure 1 illustrates that reusing of returns may increase the performance of the system. But, the determination of optimal strategies for return handling depends on the values of the cost parameters associated with the problem. Figure 2 depicts some potential strategies in the form of a decision tree. The determination of the optimal strategy for a specific product is discussed in section 4 .

At first, the collection of returns is unavoidable no matter how costly may be because the opposite 
situation will cause the customers to order and return products in order to keep them for free. So, at the first level of the decision tree we have two potential options: O1: collect returns and sell them in the secondary market and O2: collect returns and reuse as many as possible. From this first level we can see that the collection of returns is unavoidable because donation of the product to the customer will cause the customer to order and return products in order to keep them.

We must adopt $\mathrm{O} 1$ when the collection time is too long (more than the period duration) and thus, it is not possible to reuse returns. But, this may also be an option when the recovery cost is significant.

The second option $\mathrm{O} 2$ is further analyzed in $\mathrm{O} 2.1, \mathrm{O} 2.2$ and $\mathrm{O} 2.3$, according to the value and charging of the recovery cost. The option $\mathrm{O} 2.1$ is the case that the returned products can be used without any recovery, e.g. CDs where the returns are refunded only if the package is unopened. Options O2.2 and O2.3 assume that the recovery cost is significant. The difference between them is that in the first one (partial recovery) this cost is paid for every returned item we reuse, while in the second (full recovery) this cost is paid for all returns whether we reuse them or not. Finally, the recovery of returned products may incur a fixed cost paid per period (e.g., the cost of leasing a packing machine to repack returns). So in partial and full recovery options we have another branch in the tree, which includes (options O2.2.2 and O2.3.2) or not (options O2.2.1 and O2.3.1) a fixed recovery cost.

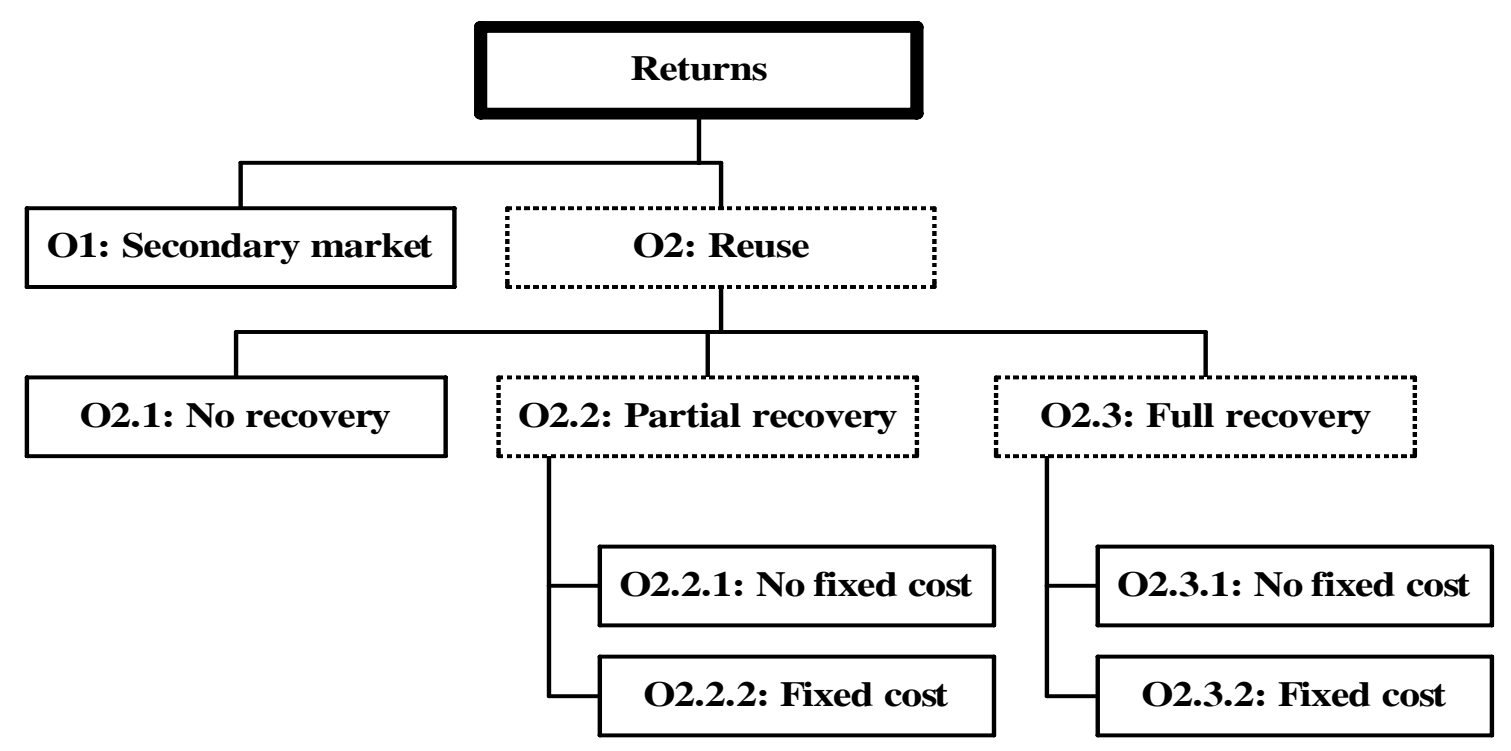

Figure 2: Options for handling returns

So, the above analysis leads to six alternative options on returns handling (O1, O2.1, O2.2.1, O2.2.2, O2.3.1 and O2.3.2). For each one of these options a model is developed. These six models are presented in section 3.3, but first, in section 3.2, we discuss our assumptions and notation. 


\subsection{Assumptions and notation}

- We remind that the length of the selling period is denoted by $T$ and the order quantity, which arrives before the beginning of the period, is denoted by $Q$. The total demand, as expressed by the customer orders, is denoted by $D$ and it is a stochastic variable with density function $f(\cdot)$ and distribution function $F(\cdot)$.

We assume that the collection and recovery time $L$, is constant. We remind that $L$ is the summation of various time delays most of which are almost fixed. A problem may be the customer's inspection time, which is quite variable for some products. In that situations we can use the proposed models to obtain an upper and a lower limit for the order quantity using the marginal values for $L$.

For the various cost parameters associated with the problem the notation of Silver et al. (1998) is followed. Specifically, let $C$ be the unit purchase cost paid to the supplier. The unit selling price is denoted by $P$ and it is assumed that $P>C$ to allow positive profit. $P$ is paid only for accepted products since a 'money back guarantee' policy forces the reimbursement of the payments for the returns. The surplus stock that remains unsold after the end of the period can be sold in the secondary market at a unit salvage value $S$. The salvage value is assumed to be less than $C$, otherwise there is no loss risk and thus, the higher the size of the initial order the higher the profit. If an order is not satisfied there is a lost sales cost denoted by $G$. This cost is included for the completeness of the analysis and it may be ignored since it is difficult to be estimated in many practical situations. Finally, the collection cost per product is denoted by $C_{c}$, the recovery cost, which is the cost to bring a returned product to an as-good-as-new condition, is denoted by $C_{r}$ and the fixed recovery cost is denoted by $C_{f}$. In order to expect a positive profit from reuse the recovery cost must be less than the profit margin for reused products, i.e. $C_{r}<P^{\prime}-S$, where $P^{\prime}$ is the expected revenue per satisfied order (see section 3.4).

The return rate is denoted by $r$ and it is assumed to be a fixed proportion of the satisfied orders. We also assume that returns are reused only once per period and this is generally valid since we use returns near the end of the selling period (after $t_{Q}$ in Figure 1) and if some of the reused products are returned again this will probably happen after the end of the period.

Another key assumption is that the fraction of returns which arrives in time to be reused is independent of the total sales. This is called serviceable return rate and it is denoted by $k(0 \leq k \leq 1)$. The estimation of $k$, which mostly depends on $L$, is not obvious. We return later in section 6 to discuss the estimation of $k$ in practical problems. Serviceable return rate $k$ is used to adjust the order quantity and provide an expression for expected profit using an analysis similar to problems without reuse. This method is often used to model returns in various reverse logistic situations and it provides quite accurate results for reasonable return rates as Van der Laan et al. (1996) and Fleischmann et al. (1997) indicate in a more general setting with returns. This assumption renders possible the analytical solutions of the models presented in the following paragraph. 
Table 1: Characteristics of model variations

\begin{tabular}{c|c|c|c|c|c|c|c}
\hline & & & Partial & Partial & Full & Fixed & \\
Model & Option & Returns & reuse & recovery & recovery & cost & Equation \\
\hline 1 & O1 & $\checkmark$ & - & - & - & - & $(2)$ \\
2 & O2.1 & $\checkmark$ & $\checkmark$ & - & - & - & $(4)$ \\
3 & O2.2.1 & $\checkmark$ & $\checkmark$ & $\checkmark$ & - & - & $(6)$ \\
4 & O2.3.1 & $\checkmark$ & $\checkmark$ & - & $\checkmark$ & - & $(8)$ \\
5 & O2.2.2 & $\checkmark$ & $\checkmark$ & $\checkmark$ & - & $\checkmark$ & $(10)$ \\
6 & O2.3.2 & $\checkmark$ & $\checkmark$ & - & $\checkmark$ & $\checkmark$ & $(12)$ \\
\hline
\end{tabular}

\subsection{Models and solutions}

- The assumptions for the six models corresponding to the return handling options of section 3.2 are summarized in Table 1 . The last column of Table 1 includes the equation numbers for the optimal order quantity of each model, which are presented in the following paragraphs. The proofs for obtaining the optimal order quantities for each model are presented in Appendix A.

Model 1 - No reuse - sell all returns in the secondary market

The expected profit $\pi_{1}(Q)$ for the first model is easily written in a way similar to the newsboy problem way:

$$
\begin{aligned}
\pi_{1}(Q)= & \int_{0}^{Q}\left[P(1-r) x+S[Q-(1-r) x]-C Q-C_{c} x\right] f(x) d x \\
& +\int_{Q}^{\infty}\left[P(1-r) Q+S r Q-G(x-Q)-C Q-C_{c} Q\right] f(x) d x
\end{aligned}
$$

Applying the first order conditions, we obtain the optimal order quantity $Q_{1}^{*}$ that maximizes (1). $Q_{1}^{*}$ is given by (2).

$$
F\left(Q_{1}^{*}\right)=\frac{(1-r) P+r\left(S-C_{c}\right)+G-C}{(1-r) P+r\left(S-C_{c}\right)+G-S}=\frac{P^{\prime}+G-C}{P^{\prime}+G-S}
$$

The optimality condition (2) is similar to the one for the classical newsboy problem, if we substitute the price $P$ with $P^{\prime}=(1-r) P+r\left(S-C_{c}\right)$, which is the expected unit revenue per satisfied order.

\section{Model 2 - Partial reuse}

The expected profit $\pi_{2}(Q)$ of the second model in now given by (3) and the optimality condition for $Q_{2}^{*}$ is given by (4). 


$$
\begin{gathered}
\pi_{2}(Q)=\int_{0}^{Q(1+k r)}\left[P^{\prime} x+S(Q-x)-C Q\right] f(x) d x+\int_{Q(1+k r)}^{\infty} P^{\prime} Q(1+k r) f(x) d x \\
+\int_{Q(1+k r)}^{\infty}[-S Q k r-G(x-Q(1+k r))-C Q] f(x) d x \\
F\left(Q_{2}^{*}(1+k r)\right)=\frac{\left(P^{\prime}+G-S\right)(1+k r)+S-C}{\left(P^{\prime}+G-S\right)(1+k r)}
\end{gathered}
$$

\section{Model 3 - Partial recovery and reuse}

In this case the expected profit $\pi_{3}(Q)$ and the optimality condition for the order quantity $Q_{3}^{*}$ are given by (5) and (6) respectively. The expected profit is the same as in model 2 reduced by two terms. The first term is the recovery cost paid if the demand is less than the maximum demand we can serve with returns $Q(1+k r)$; in this case we recover $(x-Q)$ returned products. The second term is the cost paid if demand exceeds the maximum serviceable demand; in this case we recover $(k r Q)$ returned products. In this model we assume that the salvage value $S$ can be also obtained for the returns we don't reuse even without recovery.

$$
\begin{gathered}
\pi_{3}(Q)=\pi_{2}(Q)-\int_{Q}^{Q(1+k r)} C_{r}(x-Q) f(x) d x-\int_{Q(1+k r)}^{\infty} C_{r} k r Q f(x) d x \\
\left(P^{\prime}+G-S\right)(1+k r)+S-C-C_{r} k r=\left(P^{\prime}+G-S-C_{r}\right)(1+k r) F\left(Q_{3}^{*}(1+k r)\right)+C_{r} F\left(Q_{3}^{*}\right)
\end{gathered}
$$

\section{Model 4 - Full recovery, partial reuse}

If the recovery cost is charged for all the returned products and not only for the ones we reuse as in model 3 , the expected profit function $\pi_{4}(Q)$ and the optimality condition for $Q_{4}^{*}$ are modified to $(7)$ and $(8)$ respectively. To avoid confusion we denote the full recovery cost with $C_{r}^{\prime}$. This recovery cost must also be $C_{r}^{\prime}<P^{\prime}-S$ to expect positive profit from reuse.

$$
\begin{gathered}
\pi_{4}(Q)=\pi_{2}(Q)-\int_{0}^{Q(1+k r)} C_{r}^{\prime} r x f(x) d x-\int_{Q(1+k r)}^{\infty} C_{r}^{\prime} r Q(1+k r) f(x) d x \\
F\left(Q_{4}^{*}(1+k r)\right)=\frac{\left(P^{\prime}+G-S-r C_{r}^{\prime}\right)(1+k r)+S-C}{\left(P^{\prime}+G-S-r C_{r}^{\prime}\right)(1+k r)}
\end{gathered}
$$




\section{Model 5 - Partial recovery and reuse, fixed recovery cost}

Finally, the recovery of returned products may incur a fixed cost, which is charged when the initial order quantity has depleted and we have to use returns. In this case the expected profit function $\pi_{5}(Q)$, given by (9) is equal to the expected profit for model 3 reduced by the fixed cost paid when demand is greater than the initial order quantity $Q$. A global optimality condition is difficult to obtain from (9). Equation (10) provides an optimal value for $Q_{5}^{*}$, which holds under the condition presented in Appendix A. The uniqueness of $Q_{5}^{*}$ can be proved for some distributions (e.g. normal), but not for the general distribution.

$$
\begin{gathered}
\pi_{5}(Q)=\pi_{3}(Q)-\int_{Q}^{\infty} C_{f} f(x) d x \\
\left(P^{\prime}+G-S\right)(1+k r)+S-C-C_{r} k r=\left(P^{\prime}+G-S-C_{r}\right)(1+k r) F\left(Q_{5}^{*}(1+k r)\right)+C_{r} F\left(Q_{5}^{*}\right)+C_{f} f\left(Q_{5}^{*}\right)
\end{gathered}
$$

\section{Model 6 - Full recovery, partial reuse, fixed recovery cost}

In case we have a fixed cost but with full recovery the expected profit function $\pi_{6}(Q)$ is given by (11). A global optimality condition is also difficult to be obtained from (11). Equation (12) provides an optimal value for $Q_{6}^{*}$ which holds under the condition presented in Appendix A. The uniqueness of $Q_{6}^{*}$ cannot also be proved for the general distribution.

$$
\begin{gathered}
\pi_{6}(Q)=\pi_{4}(Q)-\int_{Q}^{\infty} C_{f} f(x) d x \\
\left(P^{\prime}+G-S-r C_{r}^{\prime}\right)(1+k r)+S-C=\left(P^{\prime}+G-S-r C_{r}^{\prime}\right)(1+k r) F\left(Q_{6}^{*}(1+k r)\right)+C_{f} f\left(Q_{6}^{*}\right)
\end{gathered}
$$

\section{Decision making On haNDling RETURNS}

In section 3, plausible options for handling returns are presented as well as the respective model and solution for every option. However, in order to decide what to do with returns for a specific product, we have to estimate the associated cost parameters and compare the expected profits of the models that can be applied for this product following the decision tree in Figure 2. Although it is easy to prove that reusing returns is always preferable from selling them in a secondary market when the recovery cost is negligible (comparing models 1 and 2), the right decision is not obvious in case of significant recovery costs. However, we can write decision rules in the form of mathematical inequalities for all possible comparisons, but these are not 
so simple to apply in a practical problem without using mathematical software. For example, the decision rules for models 3 and 4 in comparison to model 1 are the following:

$$
\begin{gathered}
\pi_{3}\left(Q_{3}^{*}\right)-\pi_{1}\left(Q_{1}^{*}\right)>0 \Leftrightarrow C_{r}<\frac{\left(P^{\prime}+G-S\right) \int_{Q_{1}^{*}}^{Q^{*}(1+k r)} x f(x) d x}{\int_{Q_{3}^{*}}^{Q_{3}^{*}(1+k r)} x f(x) d x} \\
\pi_{4}\left(Q_{3}^{*}\right)-\pi_{1}\left(Q_{1}^{*}\right)>0 \Leftrightarrow C_{r}^{\prime}<\frac{\left(P^{\prime}+G-S\right) \int_{Q_{1}^{*}}^{Q_{4}^{*}(1+k r)} x f(x) d x}{r \int_{0}^{Q_{4}^{*}(1+k r)} x f(x) d x}
\end{gathered}
$$

When (13) or (14) are true it is preferable to recover and reuse returns; otherwise we sell them in a secondary market. Similar but more complicated expressions can be obtained for other models. In the following section we study, through numerical investigation, some properties of the optimal order quantities and of the associated profits.

\section{Properties of the SOlutions and numerical investigation}

Examining the optimality conditions (equations (3) to (12)), we come to some interesting properties of the solutions (for proofs see Appendix B), which are:

- In model 3 , the effect of recovery cost on the optimal order quality $Q_{3}^{*}$ depends on the sign of the following quantity:

$$
(1+k r) F\left(Q_{3}^{*}(1+k r)\right)-k r-F\left(Q_{3}^{*}\right)
$$

When this quantity is positive (negative) the optimal order quantity $Q_{3}^{*}$ is monotonically increasing (decreasing) function of $C_{r}$. We can extend this property to compare $Q_{2}^{*}$ and $Q_{3}^{*}$. Since $Q_{2}^{*}$ can be obtained from $Q_{3}^{*}$ for $C_{r}=0$, when the quantity given by (15) is positive, we have $Q_{2}^{*} \leq Q_{3}^{*}$, otherwise $Q_{2}^{*} \geq Q_{3}^{*}$. On the contrary, the expected optimal profit is always $\pi_{3}\left(Q_{3}^{*}\right) \leq \pi_{2}\left(Q_{2}^{*}\right)$.

- In model 4 , the optimal order quantity $Q_{4}^{*}$ is always a monotonically decreasing function of $C_{r}^{\prime}$. Therefore, $Q_{2}^{*} \geq Q_{4}^{*}$. Moreover, the expected optimal profit is always $\pi_{4}\left(Q_{4}^{*}\right) \leq \pi_{2}\left(Q_{2}^{*}\right)$.

- In models 5 and 6 , the optimal order quantities are less than the order quantities of the corresponding models without the fixed cost, i.e., $Q_{5}^{*}<Q_{3}^{*}$ and $Q_{6}^{*}<Q_{4}^{*}$ and the same happens for the optimal profits, i.e., $\pi_{5}\left(Q_{5}^{*}\right)<\pi_{3}\left(Q_{3}^{*}\right)$ and $\pi_{6}\left(Q_{6}^{*}\right)<\pi_{4}\left(Q_{4}^{*}\right)$.

To study some other properties of the optimal solutions that cannot be proven analytically we consider some numerical examples. In all numerical examples we assume that the demand follow a normal distribution with mean $\mu=100$ and standard deviation $\sigma=20$. The calculation of the optimal order quantities and 
Table 2: Profit loss from using standard newsboy model

\begin{tabular}{c|c|c|c|c|c|c}
\hline Profit margin & Model 1 & Model 2 & Model 3 & Model 4 & Model 5 & Model 6 \\
\hline Medium & $1.9 \%$ & $17.1 \%$ & $15.5 \%$ & $19.1 \%$ & $14.6 \%$ & $18.2 \%$ \\
High & $0.5 \%$ & $8.7 \%$ & $7.9 \%$ & $9.1 \%$ & $7.4 \%$ & $8.6 \%$ \\
\hline
\end{tabular}

expected profits for each example took a few seconds in a Pentium III PC using the mathematical software MAPLE V version 5.

At first, we calculated the improvement in total profit caused if we order the optimal order quantity of the proposed models instead of the newsboy order quantity in a problem with returns. We choose some reasonable values for the parameters, specifically $r=0.3, k=0.8, C=7, S=2, G=2, C_{c}=0.5, C_{r}=1$, $C_{f}=10$. We examined two cases: the first one with $P=14$ (medium profit margin) and the other with $P=21$ (high profit margin). Table 2 depicts the profit loss if we order the newsboy order quantity. We can see that in the case of selling the returns in a secondary market (model 1) the profit loss is rather small, but in all the cases we reuse returns the profit loss is significant, $16.8 \%$ on average for the medium profit margin and $8.3 \%$ for the high profit margin. These results also prove our statement in Section 1 that the newsboy optimal quantity is far from optimal when the return rates are high.

Hereunder, some interesting properties of the solutions are illustrated through figures. For all the case presented we assume that $P=15, G=2$ and $C_{c}=0$. Figure 3 depicts the effect of return rate $r$ and serviceable return rate $k$ to the optimal order quantity and the expected profit for a specific example assuming $C_{r}=0$. The lines for $r=0.0$ represent the optimal order quantity and the expected profit of the classical newsboy problem (without return). We observe that as return rate $(k$ or $r$ ) increases the effect on $Q^{*}$ and $\pi\left(Q^{*}\right)$ is almost linear.

Figure 4 depicts the effect of the margin of profit on optimal order quantity and expected profit of model 3. We assume a constant price $P$ and salvage value $S$ and modify unit cost $C$. When $C$ tends to $S$, the optimal order quantity tends to infinite, as explained in the beginning of section 3. Moreover, one more critical value for $C$ is the value $P^{\prime}+G-S$, for which the optimal order quantity takes its minimum value.

Figure 5 and 6 illustrate the effect of recovery cost on the optimal order quantity and expected profit of model 3 and 4 respectively, for different values of $k$. The line for $k=0.0$ represents the expected profit $\pi_{1}^{*}$ when we don't use returns (model 1). Figure 5 shows that as $C_{r}$ increases and goes to the value given by (13) (for the specific example this marginal value is $C_{r}=9.0$ ), the optimal order quantity $Q_{3}^{*}$ and the expected profit tend to the optimal order quantity and the expected profit of model 1. As Figure 6 shows, the influence of $C_{r}^{\prime}$ on the optimal order quantity and expected profit of model 4 is almost linear, quite 

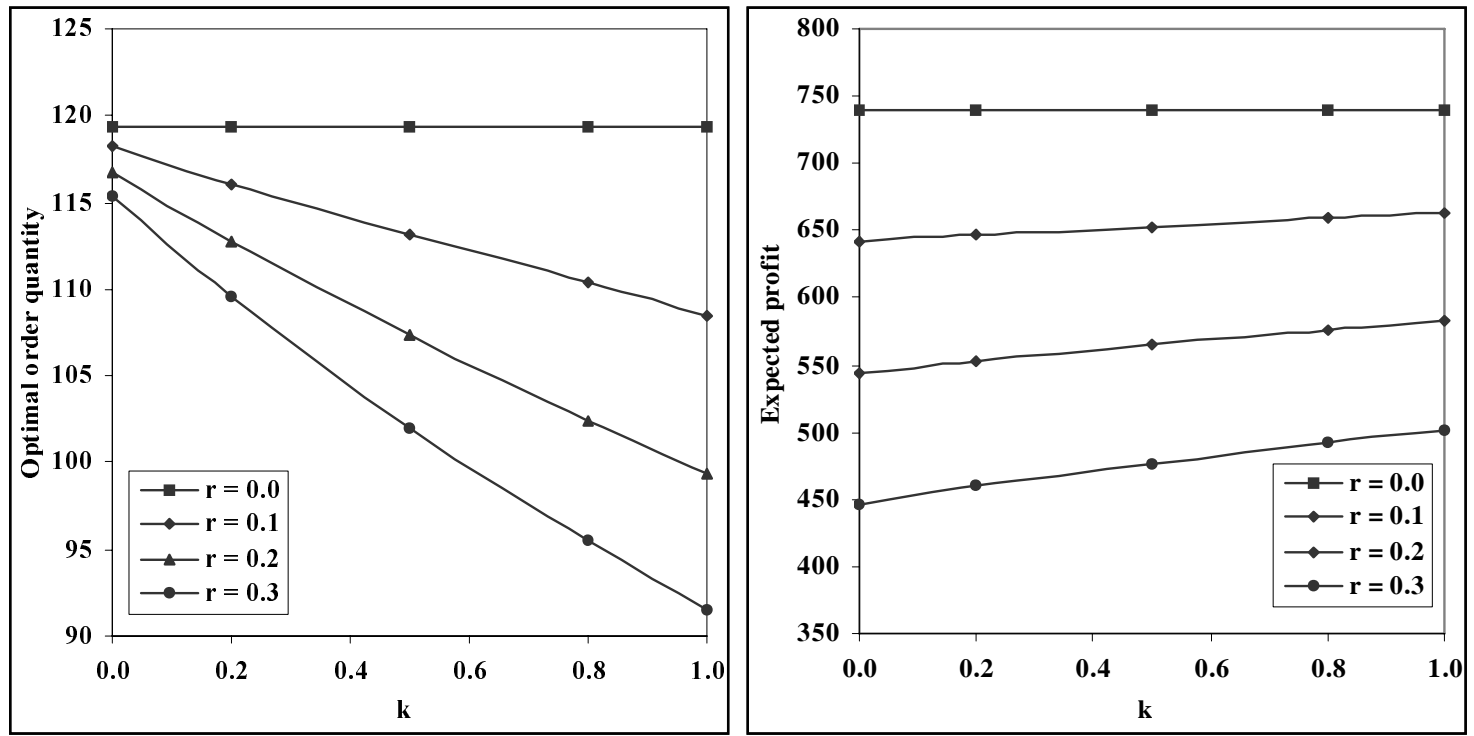

Figure 3: Effect of $r$ and $k$ on optimal order quantity $Q_{2}^{*}$ and expected profit $\pi_{2}\left(Q_{2}^{*}\right)(P=15, G=2, S=5$, $C=7)$
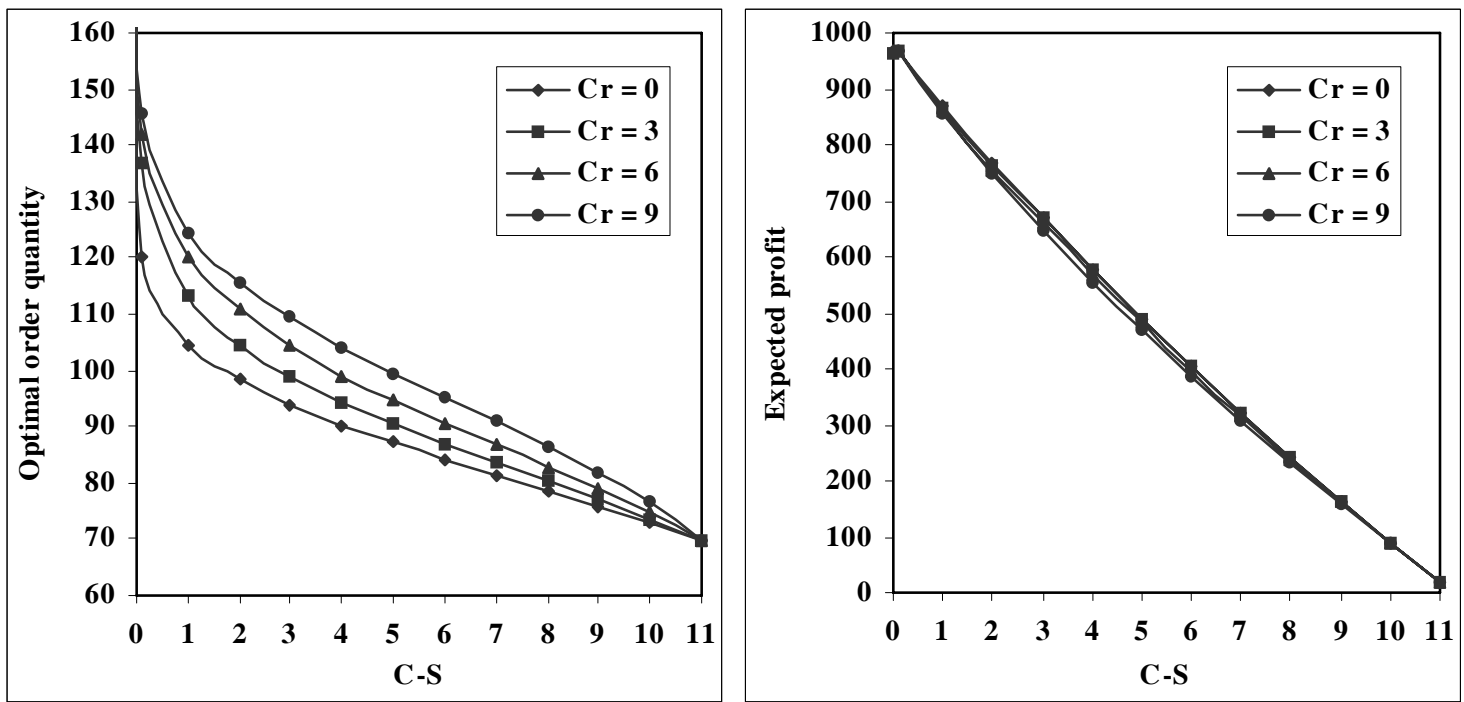

Figure 4: Effect of $(C-S)$ on optimal order quantity $Q_{3}^{*}$ and expected profir $\pi_{3}\left(Q_{3}^{*}\right)(P=15, G=2, S=1$, $r=0.3, k=0.8)$ 
different than its effect in model 3. The marginal value given by (14) are the value of $C_{r}^{\prime}$, where the profit of model 1 (line with $k=0.0$ ) cuts the profit line of the respective example.
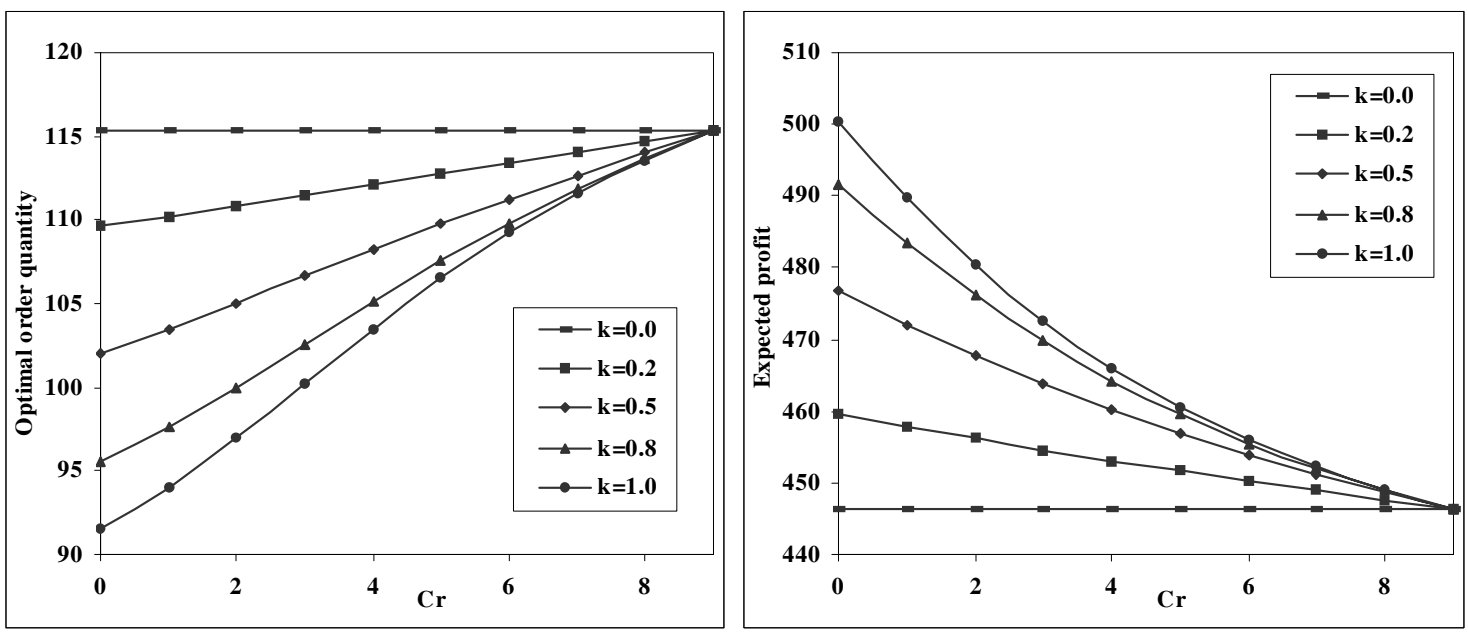

Figure 5: Effect of $C_{r}$ on the optimal order quantity $Q_{3}^{*}$ and expected profit $\pi_{3}\left(Q_{3}^{*}\right)(P=15, G=2, S=5$, $C=7, r=0.3)$
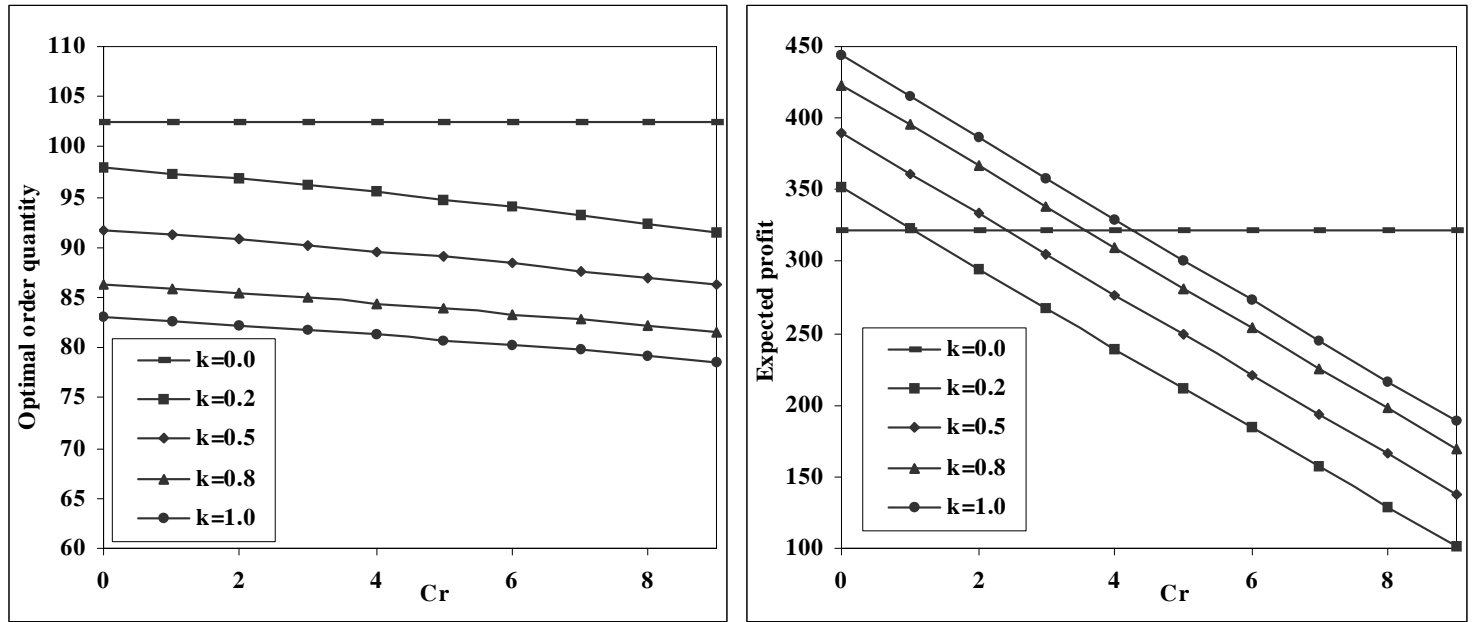

Figure 6: Effect of $C_{r}^{\prime}$ on the optimal order quantity $Q_{4}^{*}$ and expected profit $\pi_{4}\left(Q_{4}^{*}\right)(P=15, G=2, S=2$, $C=7, r=0.3)$

\section{A COMMENT On ESTimation OF SERViCEABLE RETURNS}

In section 3 , we assumed that only a part of the returns, denoted by $k$, can be used to satisfy new orders. This is true because some returns may be available close to or after the end of the selling period when the demand is low or zero and therefore by the end of the period we may have a surplus of products with only salvage value. So, to estimate $k$ we have to study the net demand, which is the difference between demand 
and returns, not cumulatively, but as a function of time. Thus, the estimation of $k$ depends on one hand on the demand distribution in time and on the other hand on the collection and recovery time $L$ of the returns. To analyze this problem further we need some additional notation.

The stochastic demand is assumed to follow a specific (for each product) pattern in time i.e., the function of demand is $D(t)=\lambda z(t)$, where $\lambda$ is a positive stochastic variable with density function $f(\cdot)$ and distribution function $F(\cdot)$ and $z(t), 0 \leq t \leq T$ is a positive function of $t$ with $\int_{0}^{T} z(t) d t=1$, which determines the pattern.

The returns, denoted by $R(t)$, are assumed to be a proportion $r$ of the associated demand, $(0 \leq r \leq 1)$. So if we assume that returned products reenter the serviceable stock after time $L$ (collection and recovery time), then the fulfillment of $D(t-L)$ orders at time $t-L$ will cause a return flow $R(t)=r \lambda z(t-L)$ at time $t$.

We also assume that the returns can be reused right after their recovery and there is no need to be stocked except for the end of the period where we may have more returns than demand. Otherwise, the analysis is more complicated since we have to include the stocked returns.

The net demand, which is the demand that is to be satisfied by the initial order, will be

$$
N(t)=\left[D(t)-\sum R(t)\right]^{+}=\lambda\left[z(t)-r z(t-L)-r^{2} z(t-2 L)-\ldots\right]^{+} \text {for } t \in[0, T]
$$

In the above expression the summation is used to describe the case that some products may be sent and returned $1,2, \ldots$ or more times. In the remaining of this section we assume that products can only be returned once per period, i.e. we ignore the terms that refer to products returned more than one times. This assumption stands in practical mail sales situations, where the collection time is usually long compared to the period length and the selling-return cycle is difficult to be repeated.

Thus, the total net demand during period $T$ will be:

$$
\begin{aligned}
\int_{0}^{T} N(t) d t & =\lambda \int_{0}^{T}[z(t)-r z(t-L)]^{+} d t=\lambda(1-k r) \\
& \Rightarrow k=\frac{1}{r} \int_{0}^{T}\left[z(t)-[z(t)-r z(t-L)]^{+}\right] d t
\end{aligned}
$$

where $k$ is a constant depending on $z(t), r$ and $L$. The lower bound for $k$ is obtained for $L \geq T$ when no returns can be reused and the upper bound is obtained for $L=0$, when all the returns are reused.

Thus, $k$ can be calculated either analytically or numerically for various distributions of the demand in time. For example if the demand pattern is uniform, i.e., $z(t)=1 / T$, for $t \in(0, T)$, then $k=\max (1-L / T, 0)$.

To study the validity of our assumptions, we estimated the serviceable return rate using some real demand data and compare it with the $k$ calculated by our model. Figure 7 depicts the demand of a single-period 
product (agenda) for four seasons starting in years 1995-1998 (Source: CERN, 1999). We observe that the length of the period is almost six months from September to February. The demand is normalized, so that the integral of demand during each one of the four periods examined is equal to 1 . We assume a return rate for the specific product equal to $r=10 \%$ and a collection and recovery time $L=1$ month. Using the real data, we can find that the fraction of the returns that could be reused, i.e. the observed serviceable return rates were $90.3 \%, 97.4 \%, 95.7 \%$ and $91.8 \%$ for the years $1995,1996,1997$ and 1998 respectively.

To model the above demand distribution in time we assume a very simple triangular demand pattern $z(t)$, which minimizes the sum of squared errors. This pattern is also depicted in Figure 7 .

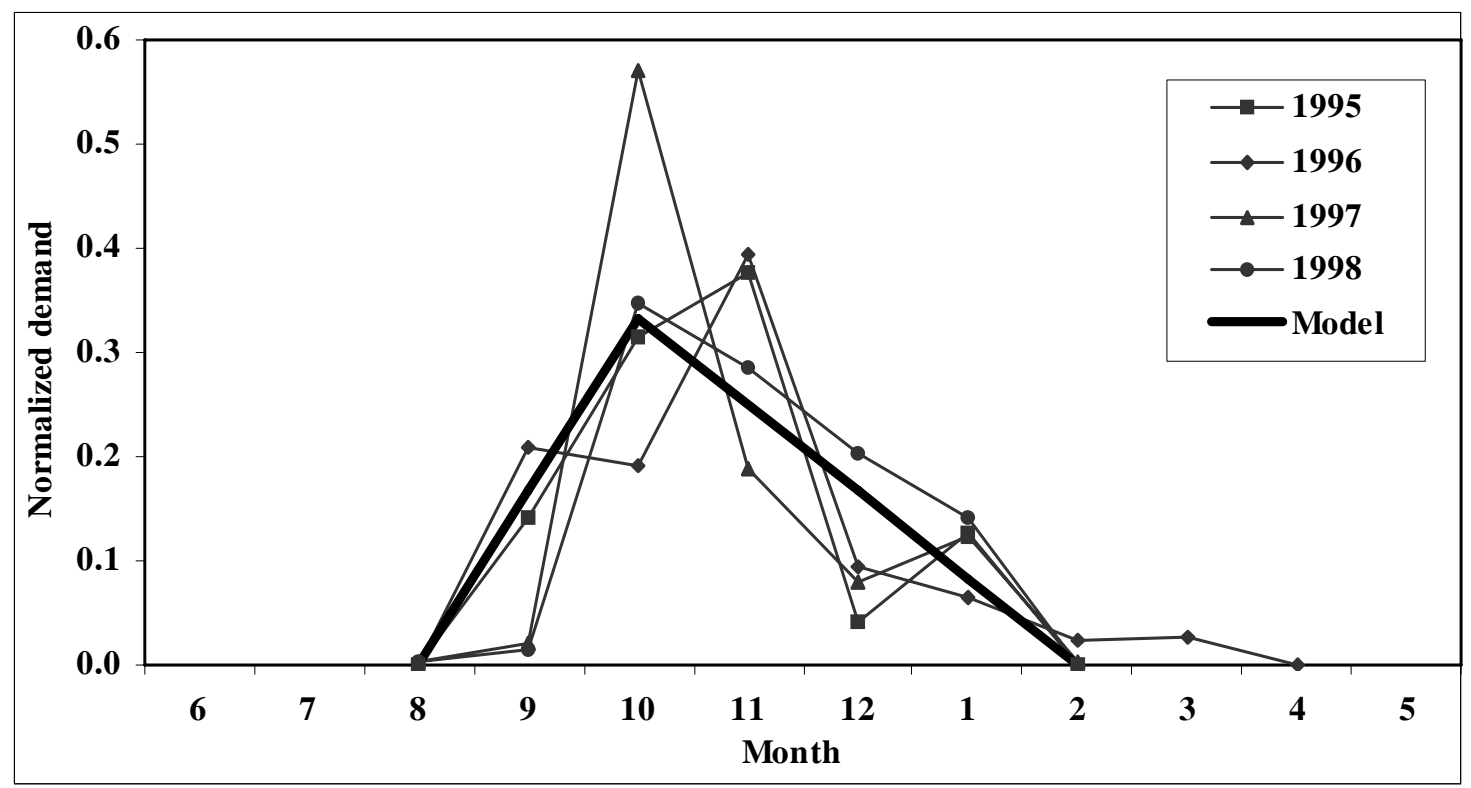

Figure 7: Actual and modeled demand pattern

Substituting $z(t)$ in (16) and solving it for $k$ we obtain $k=90.5 \%$. Then, assuming reasonable values for cost parameters we calculate the optimal expected profits using the observed and the calculated $k$. Although the differences in estimating $k$ are not negligible, the penalty in expected profit is less than $1 \%$ in every case. So, even if we use this approximate estimation for $k$ to calculate the optimal order quantity through the proposed models, we can obtain most of the profit loss we would have if we don't use the models at all.

\section{Conclusion}

In this paper we analyze the effect of return flows in estimating the initial order quantity of single-period, random demand products. We prove that when the return rate is considerable, which is more or less the case for products sold through either mail sales catalogues or e-commerce, the classical newsboy solution is inadequate. To support decision making on handling returns various alternative options are identified and studied through proper mathematical models. These models are solved under the objective of maximizing 
the expected profit. Closed form analytical expressions for the optimal order quantity are obtained. To develop the models, a 'netting' approach is followed, in which the demand is adjusted by subtracting the estimated return rate. A simple approximate method to estimate the serviceable return rate, taking into consideration the demand distribution in time, is also proposed and evaluated positively.

Future research directions include dynamic estimation of expected demand and serviceable returns (quick response) using data from the beginning of the period. This research can be combined with an improved inventory control system for single period products with returns that includes a second order during the selling period.

Acknowledgment The research presented in this paper has been sponsored by a European Union TMR network subsidy (ERB 4061 PL 97-650, REVLOG project).

Appendix A. Derivation of optimality conditions for initial order quantity

Proposition 1. The expected profit $\pi_{1}(Q)$ is concave in $Q$ and its maximum value is obtained for $Q_{1}^{*}$ given by (2).

Proof. Equation (1) yields:

$$
\begin{aligned}
\pi_{1}(Q)= & {\left[P(1-r)+\left(S-C_{c}\right) r+G-C\right] Q-G E(x) } \\
& -\left[P(1-r)+\left(S-C_{c}\right) r+G-S\right]\left[Q F(Q)-\int_{0}^{Q} x f(x) d x\right]
\end{aligned}
$$

Since

$$
\frac{\partial}{\partial Q}\left[Q F(Q)-\int_{0}^{Q} x f(x) d x\right]=F(Q)
$$

the first order condition is:

$$
\frac{\partial \pi_{1}(Q)}{\partial Q}=0 \Rightarrow P^{\prime}+G-C-\left(P^{\prime}+G-S\right) F(Q)=0
$$

The second derivative with respect to $Q$ is

$$
\frac{\partial^{2} \pi_{1}(Q)}{\partial Q^{2}}=-\left(P^{\prime}+G-S\right) f(Q)<0
$$

since

$$
P^{\prime}+G-S=(1-r)(P-S)+G>0
$$

given that $r<1$ and $P>S$. So, the optimal order quantity $Q_{1}^{*}$ is given by (A.2) which is similar to (2). 
Proposition 2. The expected profit $\pi_{2}(Q)$ is concave in $Q$ and its maximum value is obtained for $Q_{2}^{*}$ given by (4).

Proof. The expected profit $\pi_{2}(Q)$ (eq. 3) is easily transformed to:

$$
\begin{aligned}
\pi_{2}(Q)= & {\left[\left(P^{\prime}+G-S\right)(1+k r)+S-C\right] Q-G E(x) } \\
& -\left(P^{\prime}+G-S\right)\left[(1+k r) Q F(Q(1+k r))-\int_{0}^{Q(1+k r)} x f(x) d x\right]
\end{aligned}
$$

Since

$$
\frac{\partial}{\partial Q}\left[(1+k r) Q F(Q(1+k r))-\int_{0}^{Q(1+k r)} x f(x) d x\right]=(1+k r) F(Q(1+k r))
$$

the first order condition is:

$$
\begin{gathered}
\frac{\partial \pi_{2}(Q)}{\partial Q}=0 \Rightarrow \\
\left(P^{\prime}+G-S\right)(1+k r)-C-S-\left(P^{\prime}+G-S\right)(1+k r) F(Q(1+k r))=0
\end{gathered}
$$

Since the second derivative with respect to $Q$ is negative

$$
\frac{\partial^{2} \pi_{2}(Q)}{\partial Q^{2}}=-\left(P^{\prime}+G-S\right)(1+k r) f(Q(1+k r))<0
$$

the optimal order quantity $Q_{2}^{*}$ is given by (A.4) which is similar to (4).

Proposition 3. The expected profit $\pi_{3}(Q)$ is concave in $Q$ and its maximum value is obtained for $Q_{3}^{*}$ given by $(6)$.

Proof. The expected profit $\pi_{3}(Q)$ (eq. 5) is easily transformed to:

$$
\begin{aligned}
\pi_{3}(Q)= & {\left[\left(P^{\prime}+G-S\right)(1+k r)+S-C-C_{r} k r\right] Q-G E(x) } \\
& -\left(P^{\prime}+G-S-C_{r}\right)\left[(1+k r) Q F(Q(1+k r))-\int_{0}^{Q(1+k r)} x f(x) d x\right] \\
& -C_{r}\left[Q F(Q)-\int_{0}^{Q} x f(x) d x\right]
\end{aligned}
$$

The first order condition using (A.1) and (A.3) is:

$$
\begin{gathered}
\frac{\partial \pi_{3}(Q)}{\partial Q}=0 \Rightarrow\left(P^{\prime}+G-S\right)(1+k r)+S-C-C_{r} k r \\
-\left(P^{\prime}+G-S-C_{r}\right)(1+k r) F(Q(1+k r))-C_{r} F(Q)=0
\end{gathered}
$$

The second derivative with respect to $Q$ is given by

$$
\frac{\partial^{2} \pi_{3}(Q)}{\partial Q^{2}}=-\left(P^{\prime}+G-S-C_{r}\right)(1+k r) f(Q(1+k r))-C_{r} f(Q)
$$

which is always negative since

$$
C_{r}<P^{\prime}-S \Rightarrow P^{\prime}+G-S-C_{r}>0
$$

the optimal order quantity $Q_{3}^{*}$ is given by (A.5) which is similar to (6). 
Proposition 4. The expected profit $\pi_{4}(Q)$ is concave in $Q$ and its maximum value is obtained for $Q_{4}^{*}$ given by (8).

Proof. The expected profit $\pi_{4}(Q)$ (eq. 7) is easily transformed to:

$$
\begin{aligned}
\pi_{4}(Q)= & {\left[\left(P^{\prime}+G-S\right)(1+k r)+S-C-C_{r}^{\prime} r(1+k r)\right] Q-G E(x) } \\
& -\left(P^{\prime}+G-S-r C_{r}^{\prime}\right)\left[(1+k r) Q F(Q(1+k r))-\int_{0}^{Q(1+k r)} x f(x) d x\right]
\end{aligned}
$$

The first order condition using (A.3) is:

$$
\begin{gathered}
\frac{\partial \pi_{4}(Q)}{\partial Q}=0 \Rightarrow\left(P^{\prime}+G-S\right)(1+k r)+S-C-C_{r}^{\prime} r(1+k r) \\
-\left(P^{\prime}+G-S-r C_{r}^{\prime}\right)(1+k r) F(Q(1+k r))=0
\end{gathered}
$$

The second derivative with respect to $Q$ is given by

$$
\frac{\partial^{2} \pi_{4}(Q)}{\partial Q^{2}}=-\left(P^{\prime}+G-S-r C_{r}^{\prime}\right)(1+k r) f(Q(1+k r))
$$

which is always negative since

$$
r C_{r}^{\prime}<C_{r}^{\prime}<P^{\prime}-S<P^{\prime}+G-S \Rightarrow P^{\prime}+G-S-r C_{r}^{\prime}>0
$$

the optimal order quantity $Q_{4}^{*}$ is given by (A.6) which is similar to (8).

Proposition 5. The expected profit $\pi_{5}(Q)$ is maximized for $Q_{5}^{*}$ given by (10).when

$$
-\left(P^{\prime}+G-S-C_{r}\right)(1+k r) f(Q(1+k r))-C_{r} f(Q)-C_{f} \frac{\partial}{\partial Q} f(Q)<0
$$

Proof. The expected profit $\pi_{5}(Q)$ (eq. 9) is easily transformed to:

$$
\begin{aligned}
\pi_{5}(Q)= & {\left[\left(P^{\prime}+G-S\right)(1+k r)+S-C-C_{r} k r\right] Q-G E(x) } \\
& -\left(P^{\prime}+G-S-C_{r}\right)\left[(1+k r) Q F(Q(1+k r))-\int_{0}^{Q(1+k r)} x f(x) d x\right] \\
& -C_{r}\left[Q F(Q)-\int_{0}^{Q} x f(x) d x\right]+C_{f}[1-F(Q)]
\end{aligned}
$$

The first order condition using (A.1) and (A.3) is:

$$
\begin{gathered}
\frac{\partial \pi_{5}(Q)}{\partial Q}=0 \Rightarrow\left(P^{\prime}+G-S\right)(1+k r)+S-C-C_{r} k r \\
-\left(P^{\prime}+G-S-C_{r}\right)(1+k r) F(Q(1+k r))-C_{r} F(Q)-C_{f} f(Q=0
\end{gathered}
$$

When the second derivative with respect to $Q$ is negative, i.e

$$
\frac{\partial^{2} \pi_{5}(Q)}{\partial Q^{2}}=-\left(P^{\prime}+G-S-C_{r}\right)(1+k r) f(Q(1+k r))-C_{r} f(Q)-C_{f} \frac{\partial}{\partial Q} f(Q)<0
$$

the optimal order quantity $Q_{5}^{*}$ is given by (A.7) which is similar to (10). 
Proposition 6. The expected profit $\pi_{6}(Q)$ is is maximized for $Q_{6}^{*}$ given by (12) when

$$
-\left(P^{\prime}+G-S-r C_{r}^{\prime}\right)(1+k r) f\left(Q_{6}(1+k r)\right)-C_{f} \frac{\partial}{\partial Q} f\left(Q_{6}\right)<0
$$

Proof. The expected profit $\pi_{6}(Q)$ (eq. 11) is easily transformed to:

$$
\begin{aligned}
\pi_{6}(Q)= & {\left[\left(P^{\prime}+G-S\right)(1+k r)+S-C-r C_{r}^{\prime}(1+k r)\right] Q-G E(x) } \\
& -\left(P^{\prime}+G-S-r C_{r}^{\prime}\right)\left[(1+k r) Q F(Q(1+k r))-\int_{0}^{Q(1+k r)} x f(x) d x\right] \\
& +C_{f}[1-F(Q)]
\end{aligned}
$$

The first order condition using (A.3) is:

$$
\begin{gathered}
\frac{\partial \pi_{6}(Q)}{\partial Q}=0 \Rightarrow\left(P^{\prime}+G-S-r C_{r}^{\prime}\right)(1+k r)+S-C \\
-\left(P^{\prime}+G-S-r C_{r}^{\prime}\right)(1+k r) F(Q(1+k r))-C_{f} f(Q)=0
\end{gathered}
$$

When the second derivative with respect to $Q$ is negative, i.e.

$$
\frac{\partial^{2} \pi_{6}(Q)}{\partial Q^{2}}=-\left(P^{\prime}+G-S-r C_{r}^{\prime}\right)(1+k r) f(Q(1+k r))-C_{f} \frac{\partial}{\partial Q} f(Q)<0
$$

the optimal order quantity $Q_{6}^{*}$ is given by (A.8), which yields (12).

Appendix B. Properties of optimal solutions

Proposition 7. The optimal order quantity $Q_{3}^{*}$ is a monotonically increasing function of the recovery cost $C_{r}$ when

$$
(1+k r) F\left(Q_{3}^{*}(1+k r)\right)-k r-F\left(Q_{3}^{*}\right)>0
$$

Moreover, $Q_{3}^{*} \geq Q_{2}^{*}$ under the same condition and $\pi_{3}\left(Q_{4}^{*}\right) \leq \pi_{2}\left(Q_{2}^{*}\right)$.

Proof. Differentiating (A.5) that characterizes the optimal $Q_{3}^{*}$, with respect to $C_{r}$ gives:

$$
\frac{\partial A\left(Q_{3}^{*}\right)}{\partial Q_{3}^{*}} \frac{\partial Q_{3}^{*}}{\partial C_{r}^{\prime}}+\frac{\partial A\left(Q_{3}^{*}\right)}{\partial C_{r}^{\prime}}=0
$$

where $A\left(Q_{3}^{*}\right)$ is the left-hand side of $(A .5)$. The partial derivative of $A\left(Q_{4}^{*}\right)$ with respect to $Q_{3}^{*}$ is

$$
\frac{\partial A\left(Q_{3}^{*}\right)}{\partial Q_{3}^{*}}=-\left(P^{\prime}+G-S-C_{r}\right)(1+k r) f\left(Q_{3}^{*}(1+k r)\right)-C_{r} f\left(Q_{3}^{*}\right)<0
$$

for reasonable values of $C_{r}$. The partial derivative of $A\left(Q_{3}^{*}\right)$ with respect to $C_{r}$ is

$$
\frac{\partial A\left(Q_{3}^{*}\right)}{\partial C_{r}}=(1+k r) F\left(Q_{3}^{*}(1+k r)\right)-k r-F\left(Q_{3}^{*}\right)
$$

Therefore, when (A.10) is negative, (A.9) yields

$$
\frac{\partial Q_{3}^{*}}{\partial C_{r}}<0
$$


which proves that, under the above condition, the optimal $Q_{3}^{*}$ is increasing in $C_{r}$.

The optimal order quantity $Q_{2}^{*}$ is given from $Q_{3}^{*}$ when $C_{r}=0$. So, from the first part of the proposition $Q_{3}^{*} \geq Q_{2}^{*}$ when $($ A.10) is negative.

From (5) is obvious that $\pi_{3}(Q) \leq \pi_{2}(Q)$ for every $Q$ and specifically for $Q=Q_{3}^{*}$. Moreover, $\pi_{2}\left(Q_{3}^{*}\right) \leq$ $\pi_{2}\left(Q_{2}^{*}\right)$, since $Q_{2}^{*}$ is the optimal value. Therefore $\pi_{3}\left(Q_{3}^{*}\right) \leq \pi_{2}\left(Q_{2}^{*}\right)$.

Proposition 8. The optimal order quantity $Q_{4}^{*}$ is a monotonically decreasing function of the recovery cost $C_{r}^{\prime}$. Moreover, $Q_{4}^{*} \leq Q_{2}^{*}$ and $\pi_{4}\left(Q_{4}^{*}\right) \leq \pi_{2}\left(Q_{2}^{*}\right)$.

Proof. Differentiating (A.6) that characterizes the optimal $Q_{4}^{*}$, with respect to $C_{r}^{\prime}$ gives:

$$
\frac{\partial B\left(Q_{4}^{*}\right)}{\partial Q_{4}^{*}} \frac{\partial Q_{4}^{*}}{\partial C_{r}^{\prime}}+\frac{\partial B\left(Q_{4}^{*}\right)}{\partial C_{r}^{\prime}}=0
$$

where $B\left(Q_{4}^{*}\right)$ is the left-hand side of (A.6). The partial derivative of $B\left(Q_{4}^{*}\right)$ with respect to $Q_{4}^{*}$ is

$$
\frac{\partial B\left(Q_{4}^{*}\right)}{\partial Q_{4}^{*}}=-\left(P^{\prime}+G-S-r C_{r}^{\prime}\right)(1+k r) f\left(Q_{4}^{*}(1+k r)\right)<0
$$

for reasonable values of $C_{r}^{\prime}$. The partial derivative of $B\left(Q_{4}^{*}\right)$ with respect to $C_{r}^{\prime}$ is

$$
\frac{\partial B\left(Q_{4}^{*}\right)}{\partial C_{r}^{\prime}}=-r(1+k r)\left(1-F\left(Q_{4}^{*}(1+k r)\right)<0\right.
$$

Therefore, (A.11) yields

$$
\frac{\partial Q_{4}^{*}}{\partial C_{r}^{\prime}}<0
$$

which proves that the optimal $Q_{4}^{*}$ is decreasing in $C_{r}^{\prime}$.

The optimal order quantity $Q_{2}^{*}$ is given from $Q_{4}^{*}$ when $C_{r}^{\prime}=0$. So, from the first part of the proposition $Q_{4}^{*} \leq Q_{2}^{*}$

From (7) is obvious that $\pi_{4}(Q) \leq \pi_{2}(Q)$ for every $Q$ and specifically for $Q=Q_{4}^{*}$. Moreover, $\pi_{2}\left(Q_{4}^{*}\right) \leq$ $\pi_{2}\left(Q_{2}^{*}\right)$, since $Q_{2}^{*}$ is the optimal value. Therefore $\pi_{4}\left(Q_{4}^{*}\right) \leq \pi_{2}\left(Q_{2}^{*}\right)$.

Proposition 9. The effect of fixed cost is to decrease the optimal order quantity and the expected profit, i.e. $Q_{5}^{*} \leq Q_{3}^{*}, Q_{6}^{*} \leq Q_{4}^{*}$ and $\pi_{5}\left(Q_{5}^{*}\right) \leq \pi_{3}\left(Q_{3}^{*}\right), \pi_{6}\left(Q_{6}^{*}\right) \leq \pi_{4}\left(Q_{4}^{*}\right)$.

Proof. By subtracting equations (A.5) and (A.7) side by side, we obtain:

$$
\begin{gathered}
\left(P^{\prime}+G-S-C_{r}\right)(1+k r)\left[F\left(Q_{3}^{*}(1+k r)\right)-F\left(Q_{5}^{*}(1+k r)\right)\right] \\
+C_{r}\left[F\left(Q_{3}^{*}\right)-F\left(Q_{5}^{*}\right)\right]=C_{f} f\left(Q_{5}\right)>0
\end{gathered}
$$

which is true only when $Q_{5}^{*} \leq Q_{3}^{*}$.

Similarly, by subtracting equations (A.6) and (A.8) side by side, we obtain:

$$
\left(P^{\prime}+G-S-r C_{r}^{\prime}\right)(1+k r)\left[F\left(Q_{4}^{*}(1+k r)\right)-F\left(Q_{6}^{*}(1+k r)\right)\right]=C_{f} f\left(Q_{5}\right)>0
$$


which is true only when $Q_{6}^{*} \leq Q_{4}^{*}$.

From (9) is obvious that $\pi_{5}(Q) \leq \pi_{3}(Q)$ for every $Q$ and specifically for $Q=Q_{5}^{*}$. Moreover, $\pi_{3}\left(Q_{5}^{*}\right) \leq$ $\pi_{3}\left(Q_{3}^{*}\right)$, since $Q_{3}^{*}$ is the optimal value. Therefore $\pi_{5}\left(Q_{5}^{*}\right) \leq \pi_{3}\left(Q_{3}^{*}\right)$.

From (11) is obvious that $\pi_{6}(Q) \leq \pi_{4}(Q)$ for every $Q$ and specifically for $Q=Q_{6}^{*}$. Moreover, $\pi_{4}\left(Q_{6}^{*}\right) \leq$ $\pi_{4}\left(Q_{4}^{*}\right)$, since $Q_{4}^{*}$ is the optimal value. Therefore $\pi_{6}\left(Q_{6}^{*}\right) \leq \pi_{4}\left(Q_{4}^{*}\right)$.

\section{REFERENCES}

[1] Chambers, M., Eglese, R., 1986. Use of preview exercises to forecast demand for new lines in mail order, Journal of Operational Research Society 3 (3) 267-273.

[2] Chang, S.H., Fyffe D.E., 1971. Estimation of forecast errors for seasonal style-goods sales, Management Science 18 (2) B89-B96.

[3] Dekker, R., van der Laan, E. Inderfuth, K., 2000. A review on inventory control for joint manufacturing and remanufacturing, working paper.

[4] Ehrhardt, R., Taube L., 1987. An inventory model with random replenishment quantities, International Journal of Production Research 25 (12) 1795-1803.

[5] Emmons, H., Gilbert, S.M., 1998. Note. The role of returns policies in pricing and inventory decisions for catalogue goods, Management Science 44 (2) 276-283.

[6] Fisher, M., Raman, A., 1996. Reducing the cost of demand uncertainty through accurate response to early sales, Operations Research 44 (1) 87-99.

[7] Fleischmann, M., Bloemhof-Ruwaard, J.M., Dekker, R., van der Laan, E.A., van Nunen, J.A.E.E.,Van Wassenhove, L.N., 1997. Quantitative models for reverse logistics: A review, European Journal of Operational Research 103 1-17.

[8] Gentry, C.R., 1999. Reducing the cost of returns, Chain Store Age 75 (10) 124-126.

[9] Guide, V.D.R. Jr., Srivastava, R, 1998. Inventory buffers in recoverable manufacturing, Journal of Operations Management 16 (5) 551-568.

[10] Khouja, M., 1996. A note on the newsboy problem with an emergency supply option, Journal of Operational Research Society 47 1530-1534.

[11] Khouja, M., 1999. The single-period (news-vendor) problem: literature review and suggestions for future research, Omega 27 537-553. 
[12] Lau, H-S., Lau, A.H-L., 1997. A semi-analytical solution for a news boy problem with mid-period replenishment, Journal of Operational Research Society 48 1245-1253.

[13] Lau, H-S., Lau, A.H-L., 1997. Reordering Strategies for a newsboy-type product, European Journal of Operational Research 103 557-572.

[14] Meyer, H., 1999. Many happy returns, The Journal of Business Strategy 20 (4) 27-31.

[15] Murray, G., Silver, E.A., 1966. A Bayesian analysis of the style goods inventory problem, Management Science 12 (11) 785-797.

[16] Noori, A.H., Keller, G., 1986. One-period order quantity strategy with uncertain match between the amount received and quantity requisitioned, INFOR 24 (1) 1-11.

[17] Silver, E.A., Pyke, D.F., Peterson, R.P., 1998. Inventory management and production planning and scheduling, John Wiley, 3th edit.

[18] Van der Laan, E.A., Dekker, R., Salomon, M., 1996. Production planning and inventory control with remanufacturing: a numerical comparison between alternative disposal strategies, International Journal of Production Economics 46-47 339-350.

[19] Webster, S., Weng, Z.K., 2000. A risk-free perishable item returns policy, Manufacturing and Service Operations Management 2 (1) 100-106. 